Typeset using LATEX twocolumn style in AASTeX631

Draft version April 20, 2021

\title{
The Relative Importance of Faraday Rotation and QED Birefringence for the Linear Polarization of X-rays from Mass Accreting Black Holes
}

\author{
Henric Krawczynski, ${ }^{1}$ Lindsey Lisalda, ${ }^{1}$ and Charles Gammie ${ }^{2}$ \\ ${ }^{1}$ Washington University in St. Louis, Physics Department, McDonnell Center for the Space Sciences, and \\ the Center for Quantum Sensors, 1 Brookings Dr., CB 1105, St. Louis, MO 63130 \\ ${ }^{2}$ University of Urbana-Champaign, Astronomy Department, 202 Astronomy Building, 1002 W. Green Street, Physics Department, 235 \\ Loomis Lab, 1110 W. Green Street, Urbana, IL 61801
}

\begin{abstract}
The upcoming IXPE (2-8 keV) and XL-Calibur (15-75 keV) missions will make it possible to measure the linear polarization of X-rays from mass accreting stellar mass black holes with unprecedented sensitivity, enabling the accurate measurement of percent-level and in some cases even sub-percent level polarization fractions. The measurements are expected to constrain the spins, inclinations, and the structure of the accretion flows of the observed black holes. The effects of Faraday rotation and birefringence of the Quantum Electrodynamics (QED) vacuum may impact the observable polarization fractions and angles, complicating the interpretation of the results. We estimate the importance of both effects for X-rays from stellar mass and supermassive black holes and discuss the implications of the results for the upcoming IXPE and XL-Calibur observations.
\end{abstract}

Keywords: high energy astrophysics, X-ray astronomy, stellar mass black holes, supermassive black holes

\section{INTRODUCTION}

The X-ray polarimetry missions Imaging $X$-ray $P$ olarimetry Explorer (IXPE) (2-8 keV, launch in 2021) (Soffitta et al. 2020) and XL-Calibur (Abarr et al. 2021) (15-75 keV, launches in 2022 and 2023) are expected to deliver the first high-accuracy measurements of the linear polarization fractions and angles of the intermediate and hard X-rays from stellar mass black holes. For the brightest extragalactic sources, IXPE may deliver some first polarization detections. The X-ray polarimetry observations will give us two new observables (polarization fraction and angle, or Stokes $Q / I$ and $U / I$, giving the fractional intensities of the polarization along vertical/horizontal and diagonal directions, respectively) that can be used to test and refine models that were developed based on spectral (Stokes $I$ ) and timing information alone. Note that the measurement of the circular polarization (Stokes $V$ ) of the X-rays from astrophysical sources is not feasible with the currently available technologies.

The X-rays from stellar mass black holes in the thermal state are believed to come from a geometrically thin, optically thick multi-temperature accretion

Corresponding author: Henric Krawczynski, krawcz@wustl.edu disk. Detailed General Relativistic Magnetohydrodynamic (GRMHD) simulations of such systems largely validate the results from the early analytical solutions of Shakura \& Sunyaev (1973) and Novikov \& Thorne (1973); Page \& Thorne (1974) in terms of the radial temperature and luminosity profiles (Noble et al. 2011; Kulkarni et al. 2011; Zhu et al. 2012). If geometrically thin, optically thick accretion disks are indeed rather simple, then the 2-8 keV IXPE observations of the linear polarization fraction and angle of the thermal emission and the $15-75 \mathrm{keV} X L$-Calibur constraints on the polarization properties of the power law component will enable a new way of measuring the inclination (angle between the angular momentum vector of the inner accretion disk and the observer) (Li et al. 2009), and the spin of the black hole (Schnittman \& Krolik 2009; Dovčiak et al. 2012; Krawczynski 2012). The observations of the intermediate and hard states with $I X P E$ and $X L$-Calibur will make it possible to constrain the disk and corona geometry and location (Schnittman \& Krolik 2010; Schnittman et al. 2013a; Beheshtipour et al. 2017; Zhang et al. 2019). The observations will test the thin disk and corona paradigms, and are important for validating or refining black hole spin measurement methods (e.g. Brenneman 2013; Tomsick 2019; Krawczynski 2018). 
Most polarization predictions in the literature assume that the effects of Faraday rotation and conversion (Rybicki \& Lightman 1986; Huang \& Shcherbakov 2011) and Quantum Electrodynamics (QED) vacuum birefringence (Euler \& Kockel 1935; Heisenberg \& Euler 1936; Weisskopf 1936; Schwinger 1951; Toll 1952; Adler 1971; Tsai \& Erber 1975; Heyl \& Hernquist 1997; Dittrich \& Gies 1998; Hattori \& Itakura 2013; Schubert 2000; Pétri 2015; Denisov et al. 2017; Heyl \& Caiazzo 2018) on the linear polarization properties of the X-rays can be neglected. This would put X-ray polarimetry in a privileged position as Faraday rotation (proportional to $\lambda^{2}$ ) and Faraday conversion (proportional to $\lambda^{3}$ ) are known to play an important role at longer wavelengths, and QED vacuum birefringence (proportional to $1 / \lambda$ ) may impact the linear polarization properties at shorter wavelengths (Caiazzo \& Heyl 2018).

Davis et al. (2009) use detailed shearing box simulations of small segments of stellar mass black holes accretion disks to estimate the impact of Faraday rotation on the linear polarization of the thermal X-rays, and find that its effect tapers out around $2 \mathrm{keV}$. Caiazzo \& Heyl (2018) raise the possibility that QED birefringence affects the observed polarization properties. Their calculation assumes mid-disk magnetic field strengths, which may exceed those of the regions outside of the accretion disk photosphere that the X-rays traverse on their way from their emission to the observer.

In this paper, we present the first estimates of the relative importance of Faraday rotation and QED birefringence for the polarization of X-rays from mass accreting stellar mass and supermassive black holes. Our main focus is on these two effects after the emission leaves the photosphere of the accretion disk and traverses the inner region of the accretion flow where magnetic fields may be appreciable. We start the discussion focused on mass accreting stellar mass black holes. We use constraints on the Thomson optical depth and the assumption of pressure equipartition between the magnetic field and the electrons to derive estimates of the electron density $n_{\mathrm{e}}$ and the magnetic field $B$ above and below the accretion disk (Section 2). Based on these estimates, we assess the relative importance of Faraday rotation and QED birefringence (Section 3). The local equipartition between magnetic field and electron pressures may not hold in the central regions of the accretions flows, and we evaluate the impact of much stronger magnetic fields in Section 4. We conclude with a discussion of how the situation changes for Active Galactic Nuclei (AGNs), and the consequences of our findings for the upcoming $I X P E$ and $X L$-Calibur observations.

We use CGS units throughout the paper.

\section{FARADAY ROTATION AND QED BIREFRINGENCE}

Throughout this section and the next, we assume that the plasma above and below the inner accretion disk, as well as in the inner accretion disk photosphere is fully ionized. For stellar mass black holes and light elements (which make up most of the plasma) this is a good approximation. Radiation propagating through a magnetized plasma with electron density $n_{\mathrm{e}}$ and magnetic field $\mathbf{B}$ rotates the polarization direction by the angle $\Delta \theta_{\mathrm{F}}$ :

$$
\Delta \theta_{\mathrm{F}}=\frac{2 \pi e^{3}}{m_{\mathrm{e}}^{2} c^{2} \omega^{2}} \int_{0}^{d} n_{\mathrm{e}} \mathbf{B} \cdot d \mathbf{s}
$$

where $e$ is the electron charge, $m_{\mathrm{e}}$ is the mass of the electron, $c$ is the speed of light, $\omega$ is the radiation's angular frequency, and $d$ is the traversed distance, and the result is positive for a right handed circular polarization with the thumb pointing parallel to the wave vector (Rybicki \& Lightman 1986; Huang \& Shcherbakov 2011). We can compare the relative impacts of Faraday rotation and electron scattering on affecting the X-ray photons by noting that the Thomson optical depth is given by:

$$
\tau=\int_{0}^{d} n_{\mathrm{e}} \sigma_{\mathrm{T}} d s
$$

where $\sigma_{\mathrm{T}}$ denotes the Thomson cross section.

Even though the Faraday rotation measure and optical depth are very different quantities both of them have a negligible impact on the emerging radiation as long as $\theta_{\mathrm{F}} \ll 1$ and $\tau \ll 1$. The relative importance of both effects depends on the magnetic field and the photon frequency $\omega$ (or wavelength $\lambda$ ). Assuming $\mathbf{B} \cdot d \mathbf{s} \approx B d s$, and and inserting constants we get:

$$
\frac{d \theta_{\mathrm{F}} / d s}{d \tau / d s}=\frac{3}{16 \pi^{2}} \frac{\lambda^{2}}{e} B .
$$

Faraday rotation thus becomes significant relative to Thomson scattering if the ratio exceeds unity which is satisfied for:

$$
B>\frac{16 \pi^{2}}{3} \frac{e}{\lambda^{2}} \approx 1.6 \times 10^{6}\left(\frac{E_{\gamma}}{\mathrm{keV}}\right)^{2} \mathrm{G}
$$

with $E_{\gamma}$ denoting the photon energy.

The effect of vacuum birefringence depends on the strength of the magnetic field in units of the critical magnetic field:

$$
B_{\mathrm{c}}=\frac{m_{\mathrm{e}}^{2} c^{3}}{e \hbar} \approx 4.413 \times 10^{13} \mathrm{G} .
$$

For weak magnetic fields with $B \ll B_{\mathrm{c}}$, the difference of the refractive indices for the radiation polarized parallel 
and perpendicular to the plane of the ambient magnetic field $\mathbf{B}$ and the radiation's wave vector $\mathbf{k}$ is given by (Toll 1952; Chanan et al. 1979):

$$
n_{\|}-n_{\perp}=\frac{\alpha}{30 \pi}\left(\frac{B_{\perp}}{B_{\mathrm{c}}}\right)^{2}
$$

where $\alpha \approx 1 / 137$ is the fine structure constant (Zyla et al. 2020) and $B_{\perp}$ is the strength of the magnetic field $\mathbf{B}$ perpendicular to $\mathbf{k}$.

Chanan et al. (1979) discuss the implication of Equation (6) on the polarization of polarized beams for a constant magnetic field, and for magnetic fields with varying magnitudes and fixed or variable directions. The magnitude of the birefringence can be characterized by the phase difference incurred by the beams with parallel and perpendicular polarization. From Equation (6) we get with $k=\omega n / c$ :

$$
\Delta \theta_{\mathrm{QED}}=\int_{0}^{d}\left(k_{||}-k_{\perp}\right) d s=\frac{\alpha \omega}{30 \pi c} \int_{0}^{d}\left(\frac{B_{\perp}}{B_{\mathrm{c}}}\right)^{2} d s .
$$

QED birefringence is of order unity or larger for:

$$
B_{\perp}>1.6 \times 10^{8}\left(\frac{d}{2 \times 10^{7} \mathrm{~cm}}\right)^{-1 / 2}\left(\frac{E_{\gamma}}{1 \mathrm{keV}}\right)^{-1 / 2} \mathrm{G} .
$$

Where $d=2 \times 10^{7} \mathrm{~cm}$ is approximately ten times the gravitational radius of a 15 solar mass black hole. The result shows already that very strong magnetic fields are needed to render QED birefringence relevant.

Referencing the Stokes parameters such that $Q=+1$ corresponds to linear polarization direction in the $\mathbf{B}$ $\mathbf{k}$ plane, the effect of vacuum birefringence causes a rotation of $\mathbf{s}$ around the $Q$-axis, so that $U$ rotates into $V$ and vice versa. When the beam is not entirely monochromatic, and the effect of vacuum birefringence is strong $\left(\Delta \theta_{\mathrm{QED}} \gg 1\right)$, the energy dependence of the rotation angle leads to a cancellation of the net $U$ and $V$ components. If the magnetic field direction changes slowly, the adiabatic theorem in quantum mechanics implies that $Q$ referenced to the slowly rotating B-k plane stays constant, while $U$ and $V$ average to zero (see Chanan et al. (1979) and also Gnedin et al. (1978); Pavlov \& Shibanov (1979); Pavlov et al. (1979); Heyl \& Shaviv (2000)).

In the following we calculate $\theta_{\mathrm{F}}$ assuming that $\mathbf{B} \| \mathbf{k}$ holds, and $\theta_{\mathrm{QED}}$ for the case of $\mathbf{B} \perp \mathbf{k}$. In actual astrophysical sources, the direction of $\mathbf{B}$ is expected to vary along the photon trajectories, and the values that we calculate thus overestimate the expected effects. We will see that $\theta_{\mathrm{F}} \ll 1$ and $\theta_{\mathrm{QED}} \ll 1$ for good portions of the relevant parameter space. If one of the values is

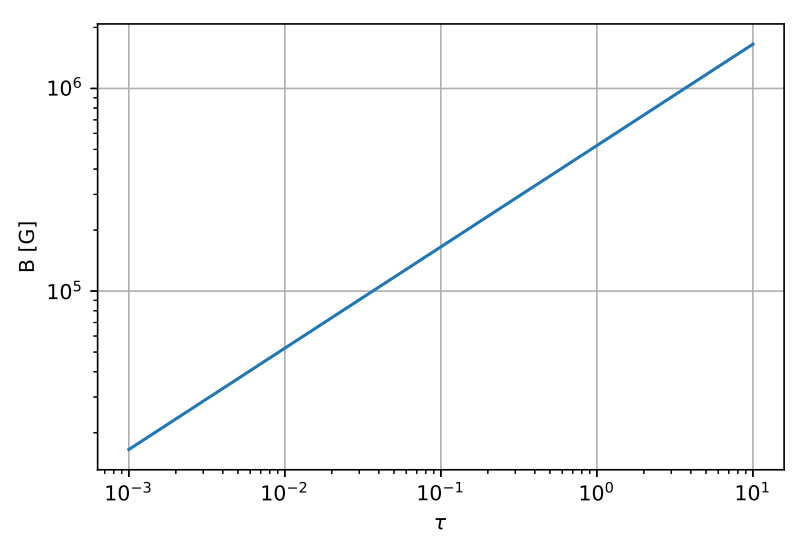

Figure 1. Equipartition magnetic field strength as function of the electron scattering optical depth $\tau$ for photons propagation over 10 gravitational radii of a 15 solar mass black hole, assuming an electron temperature of $100 \mathrm{keV}$.

comparable or much larger than 1, the combined effects of the difference in path lengths of the X-rays reaching the observer, the integration over a finite range of energies, and the expected variation of the magnetic field direction in different portions of the flow are likely to lead to a stochastic distribution of the rotation angles of different photons leading to a strong suppression of the net polarization.

\section{ESTIMATES OF THE IMPACT OF FARADAY AND QED BIREFRINGENCE ON LINEARLY POLARIZED X-RAYS}

Using the assumption that the scattering is dominated by a rather high density $n_{\mathrm{e}}(\mathbf{x}) \approx$ const over the distance $d$, we can derive a constraint on $n_{\mathrm{e}}$ from the measured electron scattering depth $\tau$ :

$$
n_{e}(\tau, d)=\frac{\tau}{d \sigma_{T}} .
$$

Assuming that the magnetic field pressure $P_{B}=B^{2} / 8 \pi$ is in equipartition with the electron pressure $P_{\mathrm{e}}=$ $n_{\mathrm{e}} k_{\mathrm{B}} T_{\mathrm{e}}$, we get a magnetic field of

$$
B\left(n_{e}, T_{e}\right)=\sqrt{8 \pi n_{e} T_{e}} .
$$

For a given $\tau, d, T_{\mathrm{e}}$, we can thus estimate $B$ and $n_{\mathrm{e}}$, and use these values to estimate $\Delta \theta_{\mathrm{F}}$ and $\Delta \theta_{\mathrm{QED}}$.

In the following, we present the results for a black hole of mass $M=15 M_{\odot}$ and a gravitational radius $r_{\mathrm{g}}=G M / c^{2}=2.2 \times 10^{6} \mathrm{~cm}(22 \mathrm{~km})$. This mass is close to that of the black holes Cyg X-1 (14.8 $\pm 1.0 M_{\odot}$, Orosz et al. 2011) and GRS $1915+105\left(12.4+2-1.8 M_{\odot}\right.$, Reid et al. 2014). 


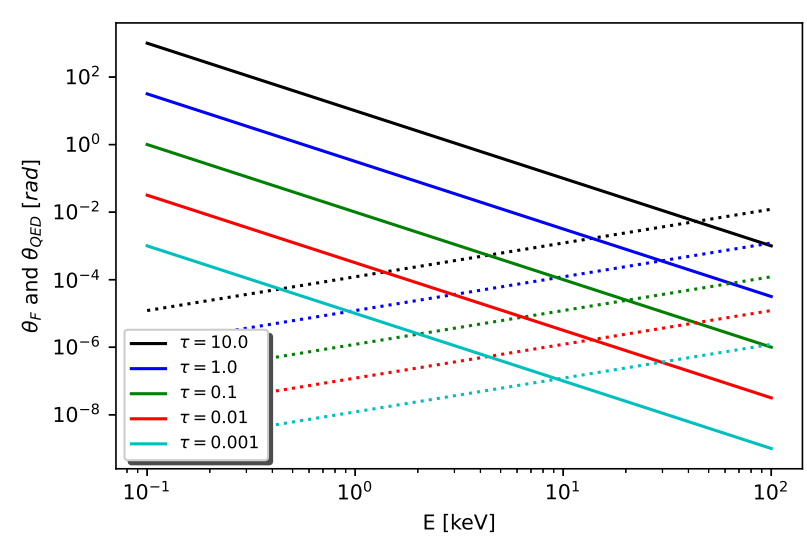

Figure 2. Faraday rotation measure $\theta_{\mathrm{F}}$ (solid lines) and QED birefringence phase difference $\theta_{\mathrm{QED}}$ between the modes parallel and perpendicular to the magnetic field (dotted lines) as a function of the photon energy. The results are shown here for the photon propagation over 10 gravitational radii of a 15 solar mass black hole, assuming an electron temperature of $100 \mathrm{keV}$, and a magnetic field in pressure equilibrium with the electrons. The color version of this figure is available online. Note for the black and white version that the optical depths decrease from top to bottom.

We consider the propagation of X-rays for different optical depths that the photons traverse before escaping from the inner accretion flow region. The photons may come from the accretion disk or the corona. Most X-ray photons originate in the central portion of the accretion flow, and we start with the assumption that the $\theta_{\mathrm{F}}$ and $\theta_{\mathrm{QED}}$ values build up over a distance $d=10 r_{\mathrm{g}}$, and that the electron temperature is $T=100 \mathrm{keV}$.

For $\tau$-values between 0.001 and 10, Equations (2-10) lead to magnetic fields between $2 \times 10^{4} \mathrm{G}$ and $2 \times 10^{6} \mathrm{G}$ (Figure 1). In the remainder of this section, we evaluate the consequences of such magnetic fields. We will discuss the impact of stronger magnetic fields in Section 4.

Figure 2 shows the implied $\theta_{\mathrm{F}}$ and $\theta_{\mathrm{QED}}$ as a function of the photon energy. Note that our assumption of a constant orientation of $\mathbf{B}$ along the path of the photons breaks down for $\tau \sim 1$ as the photons change their directions when scattering. The results are still interesting, as they constrain the relative typical strength of Faraday rotation and vacuum birefringence effects.

The figure shows that either the electron density is so low and the magnetic field is so weak that Faraday rotation and QED birefringence are both negligible, or, Faraday rotation is much stronger than QED birefringence for all energies below at least $20 \mathrm{keV}$.

Varying one parameter at a time, we get the following scaling relations: $\theta_{\mathrm{F}}(d) \propto d^{-1 / 2}$ and $\theta_{\mathrm{QED}}(d)$ is inde- pendent of $d$, and $\theta_{\mathrm{F}}\left(T_{\mathrm{e}}\right) \propto \sqrt{T_{\mathrm{e}}}$ and $\theta_{\mathrm{QED}}\left(T_{\mathrm{e}}\right) \propto T_{\mathrm{e}}$. If the X-rays run through a $100(10,000)$ times smaller region of appreciable $n_{\mathrm{e}}$ and $B$ with a similar net $\tau$ (e.g. within the photosphere of the accretion disk or in a compact wedge corona above and below the accretion disk), $\theta_{\mathrm{F}}$ would be 10 (100) times larger, and $\theta_{\mathrm{QED}}$ would not change. Faraday rotation would thus dominate even stronger over QED birefringence. For larger $d$, Faraday rotation becomes less important, and QED birefringence remains unimportant. Similarly, varying $T_{\mathrm{e}}$ from temperatures of a few $\mathrm{keV}$ all the way to $m_{e} c^{2}$ hardly changes the result.

The results above depend on the magnetic field - electron pressure equipartition assumption to estimate the magnetic field for a certain optical depth $\tau$. Dropping this hypothesis, we can derive the limiting magnetic field for which the QED birefringence phase difference increases more rapidly than the Faraday rotation measure, i.e. for which $d \theta_{\mathrm{QED}} / d s>d \theta_{\mathrm{F}} / d s$. Combining Equations (1), (7), and (9) we get:

$$
B>1.5 \times 10^{9}\left(\frac{\tau}{0.1}\right)\left(\frac{d}{2 \times 10^{7} \mathrm{~cm}}\right)^{-1}\left(\frac{E_{\gamma}}{1 \mathrm{keV}}\right)^{-3} \mathrm{G},
$$

where we used the rough estimates that $\mathbf{B} \cdot \mathrm{d} \mathbf{s} \approx B d s$ and $B_{\perp} \approx B$. This result shows that very strong magnetic fields or photon energies $>10 \mathrm{keV}$ are needed to render QED birefringence similarly or more important than Faraday rotation.

\section{DISCUSSION}

Any estimates of the magnetic field strength and electron density in the tenuous plasma above and below the accretion disk need to be taken with a grain of salt. The observed energy spectra constrain the electron scattering optical depths and densities. For stellar mass black holes, detailed GRMHD and shearing box calculations have been carried through. In the following, we compare our magnetic field estimate of $2 \times 10^{5} \mathrm{G}$ derived from the equipartition hypothesis for $\tau=0.1$ (Figure 2) with various theoretical and observational constraints.

The GRMHD simulations of Schnittman et al. (2013b) of a 10 solar mass black hole accreting at $10 \%$ of the Eddington luminosity indicate magnetic field values of between $5 \times 10^{5} \mathrm{G}$ and $5 \times 10^{6} \mathrm{G}$ in the central radiation pressure dominated region of the accretion flow along the $\tau=0.1$ contour (see their Figure 3). That is somewhat higher than the equipartition value derived above, but does not change the conclusions of our previous discussion significantly. Hirose et al. (2009) use 3-D shearing box simulations to study the vertical structure of the accretion disk of a 6.62 solar mass black hole accreting at $10 \%$ of the Eddington 
luminosity. In the inner portion of the accretion flow the radiation pressure and magnetic field pressure are in rough equilibrium and exceed the gas pressure by roughly one order of magnitude. At a radial distance of $r=30 r_{\mathrm{g}}$ from the black hole, they infer a magnetic field strength of $3 \times 10^{6} \mathrm{G}$ in the scattering photosphere (see their Figures 16 and 18). Extrapolating their results from 30 to $10 r_{\mathrm{g}}$ according to $B \propto r^{-3 / 4}$ (Shakura \& Sunyaev 1973, Equation (2.11)) gives a magnetic field of $8 \times 10^{6} \mathrm{G}$.

For a maximally spinning black hole, the accretion disk extends closer to the black hole, where the magnetic fields are stronger.

It should be noted, that none of the existing MHD studies produces the corona self-consistently, including the Compton scattering and the low Coulomb collisionality of the coronal plasma, and therefore the vertical temperature and magnetic field structure of the corona is uncertain. It is likely that magnetic reconnection will convert some of the magnetic energy of the tenuous plasma into thermal or non-thermal particle energy (see e.g. Kadowaki et al. 2018, and references therein).

We get similar results based on the $\alpha$-disk model of Shakura \& Sunyev. Based on their Equation (2.11), the mid-disk magnetic field of a 15 solar mass black hole is $\leq 6 \times 10^{7} \mathrm{G}$ at $10 r_{\mathrm{g}}$ (Shakura \& Sunyaev 1973). The result from (Hirose et al. 2009) that the magnetic field outside of the accretion disk photosphere is one order of magnitude weaker than in the disk midplane leads again to a magnetic field in the mid $10^{6} \mathrm{G}$ range.

Another magnetic field estimate can be derived from the observations of an accretion disk wind of the stellar mass black hole GRS 1915+105 (Miller et al. 2016). Assuming magnetohydrodynamic or magnetocentrifugal launching of the wind, the authors infer magnetic fields of between $10^{4} \mathrm{G}$ and $10^{6} \mathrm{G}$ at the wind launching radius of $\approx 850 \mathrm{rg}$. In the inner, radiation dominated zone of the Shakura \& Sunyaev model, the magnetic field scales proportional to $r^{-p}$ with $p=0.75$ (Shakura \& Sunyaev 1973). Further outwards, the field drops faster reaching an index of $p=1.31$ in the third zone. For $p=0.75$ the magnetic field at $10 r_{\mathrm{g}}$ would be about 30 times stronger than at $850 r_{\mathrm{g}}$. This route thus gives magnetic field values between $10^{5} \mathrm{G}$ and several times $10^{7} \mathrm{G}$. Larger $p$ values would lead to even stronger magnetic fields. Such strong magnetic fields may result from the accretion flow advecting magnetic fields from the stellar companion and may play an important role in launching collimated outflows (jets), see e.g. (Tchekhovskoy et al. 2011), and references therein.

The discussion above indicates that the X-rays may experience much stronger magnetic fields than those dis-

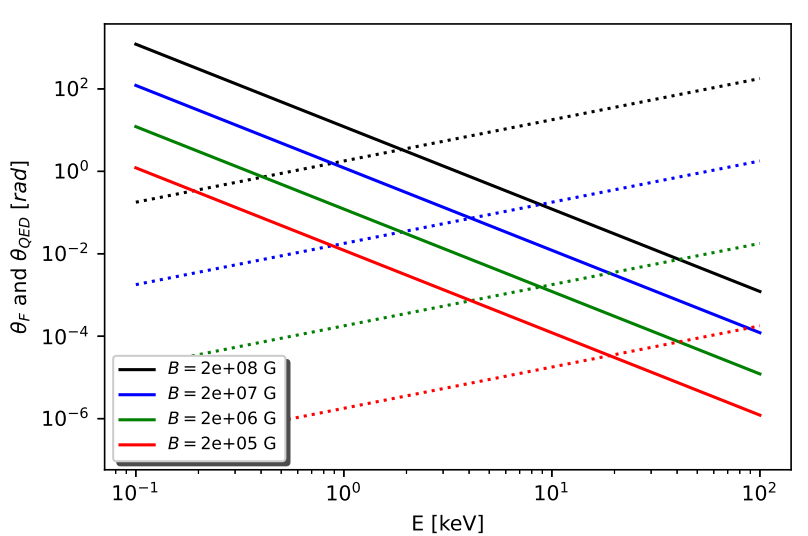

Figure 3. Faraday rotation measure $\theta_{\mathrm{F}}$ (solid lines) and QED birefringence phase difference $\theta_{\mathrm{QED}}$ for photons traversing a $10 r_{\mathrm{g}}$ large region with an electron scattering optical depth $\tau=0.1$ as a function of the magnetic field strength $B$ for a 15 solar mass black hole. The color version of this figure is available online. Note for the black and white version that the magnetic fields decrease from top to bottom.

cussed in Section 3. Figure 3 demonstrates how $\theta_{\mathrm{F}}$ and $\theta_{\mathrm{QED}}$ computed for $\tau=0.1$ change when we increase the magnetic field strength from $2 \times 10^{5} \mathrm{G}$ to values as high as $2 \times 10^{8} \mathrm{G}$. For $B=2 \times 10^{7} \mathrm{G}$, Faraday rotation dominates up to approximately $4 \mathrm{keV}$, at which QED birefringence becomes dominant. Note however, that both effects are still rather weak at this energy with $\theta_{\mathrm{F}} \approx \theta_{\mathrm{QED}} \approx 4^{\circ}$. For even stronger magnetic fields of $B=2 \times 10^{8} \mathrm{G}$, both effects become important, with QED birefringence starting to dominate over Faraday rotation at approximately $2 \mathrm{keV}$ where $\theta_{\mathrm{F}} \approx \theta_{\mathrm{QED}} \approx 180^{\circ}$.

Our discussion focused simplistically on the Faraday rotation and QED birefringence incurred over a $\sim 10 r_{\mathrm{g}}$ distance close to the black hole. We assumed that $n_{\mathrm{e}}$ drops quickly as the photons propagate away from the black hole, rendering the contribution of large distances to $\theta_{\mathrm{F}}$ unimportant. Although QED birefringence is independent of $n_{\mathrm{e}}$, it depends quadratically on $B$, so that the net contribution of large distances to $\theta_{\mathrm{QED}}$ may also be negligible. For regions far way from the central flow, it becomes increasingly unlikely that the angles between $\mathbf{B}$ and $\mathbf{k}$ does not change along the photons' trajectories, reducing the maximum possible values of $\theta_{\mathrm{F}}$ and $\theta_{\mathrm{QED}}$.

We can summarize the discussion by noting that Faraday rotation and QED birefringence, are rather weak for large regions of the likely parameter space of mass accreting stellar mass black holes. Extremely strong magnetic fields on the order of $10^{8} \mathrm{G}$ - which may be required to launch jets or winds - can lead to strong Faraday rota- 
tion and QED birefringence effects. For such magnetic fields, both effects could compete for dominance right in $I X P E$ 's $2-8 \mathrm{keV}$ energy range. The likely net effect of both effects would be a reduction of the observed linear polarization fractions. Depolarization owing to QED birefringence might be responsible for the $<8.6 \%$ upper limit on the polarization fraction of the $18 \mathrm{keV}-181 \mathrm{keV}$ emission from the stellar mass black hole Cyg X-1 reported by the $P O G O+$ team (Chauvin et al. 2018).

How does the situation change for supermassive black holes? For a $10^{9}$ solar mass black hole, 10 gravitational radii correspond to a distance of $1.48 \times 10^{15} \frac{M}{10^{9} M_{\odot}} \mathrm{cm}$. From Equation (8) we infer that QED birefringence can play a role for:

$$
B_{\perp}>1.8 \times 10^{4}\left(\frac{d}{1.5 \times 10^{15} \mathrm{~cm}}\right)^{-1 / 2}\left(\frac{E_{\gamma}}{1 \mathrm{keV}}\right)^{-1 / 2} \mathrm{G} .
$$

Equation (11) implies that QED birefringence can dominate over Faraday rotation for:

$$
B>201\left(\frac{\tau \times 1.5 \times 10^{15} \mathrm{~cm}}{d}\right)\left(\frac{E_{\gamma}}{1 \mathrm{keV}}\right)^{-3} \mathrm{G} .
$$

Equation (12) sets the more stringent requirement.

The accretion disks of AGNs are able to produce $\sim 10^{4}$ G midplane magnetic fields (Thorne et al. 1986).
Again, as for stellar mass black holes, the strength of the QED birefringence effect will depend on how the disk magnetic fields extend into the region outside of the disk, the presence of advected magnetic fields, and the orientation and uniformity of the magnetic field.

If Faraday rotation or QED birefringence modify the $\mathrm{X}$-ray polarization from stellar mass and supermassive black holes strongly, it will be challenging to disentangle the effects of the emission and reflection processes from the effects of the propagation of the X-rays through the magnetized plasma and QED vacuum. For observationally well constrained black holes, simultaneous broadband spectroscopic observations with missions like NICER and NUSTAR and broadband spectropolarimetric observations with missions like IXPE and XL-Calibur can be used to constrain the geometry of the accretion flow based on the spectral information, and the properties of the plasma and vacuum through which the Xrays propagate based on the polarization information (see also Krawczynski et al. 2019; Jahoda et al. 2019).

\section{ACKNOWLEDGEMENTS}

The authors thank an anonymous referee for very helpful comments, NASA for the support through the grants NNX16AC42G and 80NSSC18K0264, and NSF grants AST 17-16327 and AST 20-07936.

\section{REFERENCES}

Abarr, Q., Awaki, H., Baring, M. G., et al. 2021, Astroparticle Physics, 126, 102529, doi: 10.1016/j.astropartphys.2020.102529

Adler, S. L. 1971, Annals of Physics, 67, 599, doi: 10.1016/0003-4916(71)90154-0

Beheshtipour, B., Krawczynski, H., \& Malzac, J. 2017, ApJ,, 850, 14, doi: 10.3847/1538-4357/aa906a

Brenneman, L. 2013, Measuring the Angular Momentum of Supermassive Black Holes (Springer), doi: 10.1007/978-1-4614-7771-6

Caiazzo, I., \& Heyl, J. 2018, Physical Review D, 97, doi: 10/gfz5jz

Chanan, G. A., Novick, R., \& Silver, E. H. 1979, ApJ,, 228, L71, doi: 10.1086/182906

Chauvin, M., Florén, H. G., Friis, M., et al. 2018, Nature Astronomy, 2, 652, doi: 10.1038/s41550-018-0489-x

Davis, S. W., Blaes, O. M., Hirose, S., \& Krolik, J. H. 2009, The Astrophysical Journal, 703, 569, doi: 10/cm3x5k

Denisov, V. I., Dolgaya, E. E., \& Sokolov, V. A. 2017, J. High Energ. Phys., 2017, 105, doi: 10/gdwp6b
Dittrich, W., \& Gies, H. 1998, in Frontier Tests of QED and Physics of the Vacuum, ed. E. Zavattini, D. Bakalov, \& C. Rizzo, 29. https://arxiv.org/abs/hep-ph/9806417

Dovčiak, M., Muleri, F., Goosmann, R. W., Karas, V., \& Matt, G. 2012, in Journal of Physics Conference Series, Vol. 372, Journal of Physics Conference Series, 012056, doi: 10.1088/1742-6596/372/1/012056

Euler, H., \& Kockel, B. 1935, Naturwissenschaften, 23, 246, doi: 10.1007/BF01493898

Gnedin, Y. N., Pavlov, G. G., \& Shibanov, Y. A. 1978, Soviet Astronomy Letters, 4, 117

Hattori, K., \& Itakura, K. 2013, Annals of Physics, 330, 23, doi: $10 /$ f4ptcd

Heisenberg, W., \& Euler, H. 1936, Zeitschrift fur Physik, 98, 714, doi: 10.1007/BF01343663

Heyl, J., \& Caiazzo, I. 2018, arXiv:1802.00358 [astro-ph]. https://arxiv.org/abs/1802.00358

Heyl, J. S., \& Hernquist, L. 1997, Journal of Physics A: Mathematical and General, 30, 6485, doi: 10/fn9s38

Heyl, J. S., \& Shaviv, N. J. 2000, MNRAS, , 311, 555, doi: 10.1046/j.1365-8711.2000.03076.x 
Hirose, S., Krolik, J. H., \& Blaes, O. 2009, ApJ,, 691, 16, doi: 10.1088/0004-637X/691/1/16

Huang, L., \& Shcherbakov, R. V. 2011, MNRAS,, 416, 2574, doi: 10.1111/j.1365-2966.2011.19207.x

Jahoda, K., Krawczynski, H., Kislat, F., Marshall, H., \& Okajima, T. 2019, in Bulletin of the American Astronomical Society, Vol. 51, 181

Kadowaki, L. H. S., De Gouveia Dal Pino, E. M., \& Stone, J. M. 2018, ApJ,, 864, 52, doi: 10.3847/1538-4357/aad4ff

Krawczynski, H. 2012, ApJ, 754, 133, doi: 10.1088/0004-637X/754/2/133

-. 2018, General Relativity and Gravitation, 50, 100, doi: 10.1007/s10714-018-2419-8

Krawczynski, H., Matt, G., Ingram, A. R., et al. 2019, $B A A S,, 51,150$

Kulkarni, A. K., Penna, R. F., Shcherbakov, R. V., et al. 2011, MNRAS, 414, 1183, doi: 10.1111/j.1365-2966.2011.18446.x

Li, L.-X., Narayan, R., \& McClintock, J. E. 2009, ApJ, 691, 847, doi: 10.1088/0004-637X/691/1/847

Miller, J. M., Raymond, J., Fabian, A. C., et al. 2016, ApJ, 821, L9, doi: 10.3847/2041-8205/821/1/L9

Noble, S. C., Krolik, J. H., Schnittman, J. D., \& Hawley, J. F. 2011, ApJ,, 743, 115, doi: $10.1088 / 0004-637 \mathrm{X} / 743 / 2 / 115$

Novikov, I. D., \& Thorne, K. S. 1973, in Black Holes (Les Astres Occlus), 343-450

Orosz, J. A., McClintock, J. E., Aufdenberg, J. P., et al. 2011, ApJ,, 742, 84, doi: 10.1088/0004-637X/742/2/84

Page, D. N., \& Thorne, K. S. 1974, ApJ,, 191, 499, doi: 10.1086/152990

Pavlov, G. G., \& Shibanov, I. A. 1979, Zhurnal Eksperimentalnoi i Teoreticheskoi Fiziki, 76, 1457

Pavlov, G. G., Shibanov, I. A., \& Gnedin, I. N. 1979, ZhETF Pisma Redaktsiiu, 30, 137

Pétri, J. 2015, MNRAS, 451, 3581, doi: 10.1093/mnras/stv1185

Reid, M. J., McClintock, J. E., Steiner, J. F., et al. 2014, ApJ, , 796, 2, doi: 10.1088/0004-637X/796/1/2
Rybicki, G. B., \& Lightman, A. P. 1986, Radiative Processes in Astrophysics (Wiley-VCH)

Schnittman, J., Angelini, L., Baring, M., et al. 2013a, arXiv e-prints, arXiv:1301.1957.

https://arxiv.org/abs/1301.1957

Schnittman, J. D., \& Krolik, J. H. 2009, ApJ,, 701, 1175, doi: 10.1088/0004-637X/701/2/1175

-. 2010, ApJ,, 712, 908, doi: $10.1088 / 0004-637 \mathrm{X} / 712 / 2 / 908$

Schnittman, J. D., Krolik, J. H., \& Noble, S. C. 2013b, ApJ, , 769, 156, doi: 10.1088/0004-637X/769/2/156

Schubert, C. 2000, Nuclear Physics B, 585, 407, doi: $10 /$ fvc 2 jx

Schwinger, J. 1951, Physical Review, 82, 664, doi: 10.1103/PhysRev.82.664

Shakura, N. I., \& Sunyaev, R. A. 1973, A\& A,, 500, 33

Soffitta, P., Attinà, P., Baldini, L., et al. 2020, in Society of Photo-Optical Instrumentation Engineers (SPIE) Conference Series, Vol. 11444, SPIE, 1144462, doi: 10.1117/12.2567001

Tchekhovskoy, A., Narayan, R., \& McKinney, J. C. 2011, Monthly Notices of the Royal Astronomical Society: Letters, 418, L79, doi: 10.1111/j.1745-3933.2011.01147.x Thorne, K. S., Price, R. H., \& MacDonald, D. A. 1986, Black holes: The membrane paradigm

Toll, J. S. 1952, PhD thesis, PRINCETON UNIVERSITY. Tomsick, J. A. 2019, Mem. Soc. Astron. Italiana,, 90, 174

Tsai, W.-Y., \& Erber, T. 1975, Phys. Rev. D,, 12, 1132, doi: 10.1103/PhysRevD.12.1132

Weisskopf, V. 1936, Kgl. Dan. Vid. Selsk. Mazt-fys. Medd, 24,3

Zhang, W., Dovčiak, M., \& Bursa, M. 2019, ApJ,, 875, 148, doi: 10.3847/1538-4357/ab1261

Zhu, Y., Davis, S. W., Narayan, R., et al. 2012, MNRAS,, 424, 2504, doi: 10.1111/j.1365-2966.2012.21181.x

Zyla, P., et al. 2020, PTEP, 2020, 083C01, doi: $10.1093 /$ ptep/ptaa104 